\title{
Improved Ant Colony Algorithm in Logistics Time Optimal Path Selection based on the Positive and Negative Feedback and Neighboring Rights
}

\author{
Xiaochuan Guo
} China University of Geosciences (Wuhan) Mechanical and Electronic Engineering, School of
Communication Hubei province Wuhan 430074

843990360@qq.com

\begin{abstract}
Keywords: Ant colony algorithm; Connection weights; Negative feedback; Path selection
\end{abstract}
\begin{abstract}
Ant colony algorithm is a new heuristic optimization algorithm suitable for solving complex combinatorial optimization problems. Traditional ant colony algorithm converges slowly, and falls into local optimal solution, this paper presents a negative feedback connection weights improved ant colony algorithm. On the basis of the core to the ant colony algorithm, according to the selection result and continue to function through a negative feedback to adjust the connection weights, in order to achieve optimal search algorithm. In this paper, the choice of the path Yuan tong Express, for example, to the shortest path is selected as the objective function, the optimization functions by negative feedback connection weight, effectively improve the convergence speed and overall performance, it can better solve the optimal path of express logistics the selection problem.
\end{abstract}

\section{Introduction}

With the rapid growth of the national economy and social needs increasing, the original engage in mass storage, transportation logistics management activities become ever complicated in the case of product diversification and small quantities of. Logistics system is a network system, a network entity from the logistics route and logistics nodes, where in the stream line to assume an important role in the transport of goods. In the Optimization of logistics and distribution path, using suitable methods, the use of appropriate technology, formulate a reasonable distribution path, you can speed up the response to demand of customer, reduce service provider distribution operating costs and enhance customer satisfaction with the logistics system to increase economic efficiency, improving service quality. And the most important of which is the time logistics path selection problems. So, how to choose the path of logistics problems characteristic structure simple operation, excellent performance optimization heuristic, not only for the distribution system and for many can be converted to choose the path problem solving combinatorial optimization problems are great significance.

\section{Algorithm Selection}

At colony algorithm is an evolutionary algorithm to handle complex optimization problems, it is an intelligent optimization algorithm bionic groups to produce the optimal solution by simulating the behavior of ants search for food. When ants find food, they can always find an optimal path from food to nest between. Ant colony algorithm main features are: positive feedback, distributed computing, better able to blend with the other heuristics. Positive feedback process so that the method can quickly find a good solution; distributed computing makes the process easy to implement parallel; combined with other heuristic algorithms makes the method easy to find a good solution.

In this paper, to solve the problem of path optimization of logistics The basic idea is: On the basis of ant colony algorithm for solving logistics problems on the path selection, path selection based on logistics optimization problem specific circumstances, the use of ant colony algorithm as a logistics route selected core algorithm, and against ants swarm connection weights positive or negative feedback regulation, effective in improving the convergence rate of ant colony algorithm, 
convergence time and overall performance, and select a logistics route between different cities were tested to obtain better results.

\section{Introduce Improved Ant Colony Algorithm based on the Positive and Negative Feedback Neighboring Rights}

Introduction and Basic Ant Colony Algorithm Processes. Ant colony algorithm related variables as follows: $\mathrm{m}$ is the number of ants; quantity $\tau_{i j}(\mathrm{t})$ at time $\mathrm{t}$ residual edge $(i, j)$ on the arc of pheromones, the initial value of $0 ;{ }^{P_{i j}{ }^{k}}$ probability that ant $k$ from the position $i$ to $j ; C_{i j}$ for the location $i$ and $j ; \eta_{i j}$ is arc edge $(i, j)$ on the visibility, wherein $\eta_{i j}=1 / C_{i j} ; \alpha$ between the position of a consideration of the relative importance of the trajectory $(\alpha \geq 0) ; \beta$ is the relative importance of visibility ( $\beta \geq 0) ; \rho$ as a persistent trajectory $(0 \leq \rho \leq 1), 1-\rho$ construed as volatility trajectories; $N_{i}{ }^{k}$ is ant $\mathrm{k}$ at the position $i_{\text {feasible set of vertices; }} \Delta \tau_{i j}{ }^{k}$ Represents ant k in this cycle in edge arc $(i, j)$ on the left by the amount of information; ${ }^{\Delta \tau_{i j}}$ represents the number of ants on the side of the arc $(i, j)$ on the left pheromones; $L_{k}$ represents the ants are moving in this cycle path the total length; the $Q_{\text {constant. }}$

Ant colony algorithm related processes:

(1) Transfer path rule. Initially, the ants were randomly placed in a position in which the path configured according to certain transfer probability, ant $\mathrm{K}$ at time $\mathrm{t}$ transition probabilities from position $i$ to $j$ position is:

$$
P_{i j}^{k}=\frac{\left(\tau_{i j}(\mathrm{t})\right)^{\alpha}\left(\eta_{i j}\right)^{\beta}}{\sum_{u \in J_{k}}\left(\tau_{i u}(\mathrm{t})\right)^{\alpha}\left(\eta_{i u}\right)^{\beta}}
$$

Each ant according $P_{i j}{ }^{k}$ calculate the probability of selecting a path resulting from (1) can be seen, along with $\tau_{i j} P_{i j}{ }^{k}$ increases increase with $C_{i j}$ of decreases; and in accordance with the random number generated by the program, the decision under step directions, until the completion of the path traveled.

(2) Track updating rules. In each iteration, the ants move every step of the need to use local updating rule to update the pheromone concentration corresponding path; when all the ants are traversed $\mathrm{N}$ nodes, using the information of the concentration of global updating rule update path.

(3) Local update rule: Whenever all the ants after the cycle are completed, information on each path according to the formula (2) is updated, that is:

$$
\tau_{i j}(\mathrm{t}+1)=(1-\rho) \tau_{i j}(\mathrm{t})+\Delta \tau_{i j}(\mathrm{t}, \mathrm{t}+1)
$$

among them:

$$
\begin{aligned}
& \Delta \tau_{i j}(\mathrm{t}, \mathrm{t}+1)=\sum_{k=1}^{m} \Delta \tau_{i j}{ }^{k}(\mathrm{t}, \mathrm{t}+1) \\
& \Delta \tau_{i j}{ }^{k}(\mathrm{t})=Q / L_{k}
\end{aligned}
$$

(4)Global Update Rule: After all ants complete the cycle, according to the information on the path (5) to be updated, that is: 
$\tau_{i j}(\mathrm{t}+\mathrm{n})=(1-\rho) \tau_{i j}(\mathrm{t})+\Delta \tau_{i j}(\mathrm{t}, \mathrm{t}+\mathrm{n})$

among them:

$$
\Delta \tau_{i j}(\mathrm{t}, \mathrm{t}+\mathrm{n})=\sum_{k=1}^{m} \Delta \tau_{i j}{ }^{k}(\mathrm{t}, \mathrm{t}+\mathrm{n})
$$

Basic flowcharting ant colony algorithm can be summarized as shown in Fig. 1;

(1)Initialization A ( $\mathrm{t})$ \{Initialization ACO $\}$

(2)Evaluation A ( $t$ ) \{Do a fitness assessment of each ant in accordance with the objective function \}

(3)Pheromone A According to fitness, ants path through which a certain percentage of pheromone. The higher the degree of adaptation, the more pheromone released $\}$

(4)Ants Mobile \{Ant ants under the previous left pheromones and their own judgment to select the path

(5)Volatile pheromone $\{$ Pheromones will dissipate over time

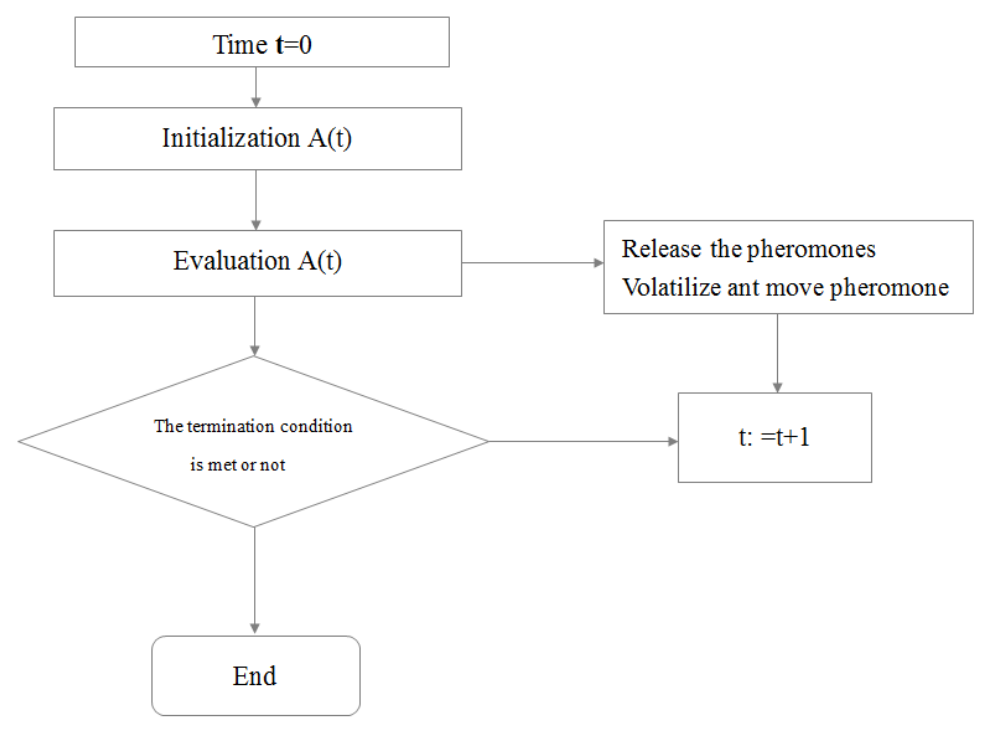

Figure 1. Ant colony algorithm flowchart

Constructed Adjacent to the Right of the Positive and Negative Feedback based on Ant Colony. Although the ant colony algorithm to narrow the search problem solving, reducing the complexity of the problem, to obtain optimum stream path, but compared with other algorithm, which generally requires a longer search time, each ant circuit constructed only feasible solution composition parts, each consisting of ants may construct circuits feasible solution, but it may not find a feasible solution, which appears after stagnation (stagnation behavior), namely the search to a certain extent, all individual solutions found exactly the same solution cannot further search space, is not conducive to find better solutions.

When the search space is small, it is difficult to search for the optimal solution, and to increase the search space to improve the search for the optimal solution to the probability of the machine number of operations will increase rapidly.

Ant colony algorithm to solve these problems, the paper chooses neighboring rights of positive and negative feedback to improve the ant colony algorithm.

Ant colony algorithm, the feedback positive feedback and negative feedback division two, the former to act now to strengthen future behavior, which now acts to undermine future behavior. Single positive feedback and negative feedback systems are unable to achieve self-organization. Self-renewal and improve the system, we need positive feedback and negative feedback combination. 
For ant colony, the negative feedback is the accumulation of pheromone $\tau$. See the formula (4), ants randomly completed a cycle, for a shorter path discovery, the legacy will be more pheromone. Then by the formula (1), the more pheromones to attract ants choose the next cycle, the higher the probability that path. Thus, the continuous cycle, ultimately, all the ants will choose the path of gradual convergence range ant colony.

Similarly, the feedback is positive colony random search technology and pheromone evaporation technology. See formula (1), the procedure is in accordance with the ants construct solutions of probability, even if the probability of selecting a path of large ants may still choose another path. Referring again to Eq.2, Eq.5, after each cycle, the pheromone on the path to be forced volatile, so as not to focus too quickly on a path. Eventually, the ant colony dispersed on each path, constantly expanding the scope of ant populations.

\section{To Improve the Logistics Path Colony Algorithm Selection based on the Construction Process of Positive and Negative Feedback}

Characterized by Entropy Pheromone Uncertainty. Entropy is the best measure of the uncertainty of the system, entropy to characterize the uncertainty of the path pheromone $\tau_{i j}$. First pheromone normalized:

$$
P_{i j}=\frac{\tau_{i j}(\mathrm{t})}{\sum_{(i, j) \in R} \tau_{i j}(\mathrm{t})}
$$

Where: The proportion accounted for a total volume of information on behalf of all the paths ${ }_{R} P_{i j}$ path $(i, j)$ on the upper, the entropy $\mathrm{S}$ can be obtained by the following formula, then the information entropy can be obtained by the following formula:

$$
S=-\sum_{(i, j) \in R} P_{i j} \operatorname{In} P_{i j}
$$

When the system is initialized, the amount of information on all paths are constant, this time $S=\operatorname{Smax}=\operatorname{In}[n(n-1) / 2]$, corresponding to the positive feedback largest state; With arithmetic operation, the entropy decreases, reducing the corresponding positive feedback, negative feedback enhanced status; Finally, when the algorithm converges only on the amount of information there is also a path, and this path all the information sub-paths are almost equal, and therefore $S=\operatorname{Smin}=\operatorname{In} n$ corresponds to the negative feedback the maximum.

Visible, $\mathrm{S}$ is good reflection of the strength of positive and negative feedback system changes. Parameters used $S$ to guide the adaptive change is reasonable. In order to facilitate the calculation of formula (8) values are mapped to the interval $[0,1]$ to obtain a correction of information entropy.

$$
E=(S-\operatorname{Inn}) /\{\operatorname{In}[n(n-1) / 2]-\operatorname{In} n\}
$$

During this algorithm runs and the $E$ is from 1 down to 0 , to facilitate subsequent parameters adaptive design [7].

Information Entropy and Algorithm State. First, the algorithm in the early, mid or late, according to the $\mathrm{E}(\mathrm{t})$ the size of the discriminated. Custom algorithm pre - Interim Pro Tpm critical point (take 0.7 ), the mid - late critical point Tma (preferably 0.3 ), it is considered $0.7<\mathrm{E}(\mathrm{T})<1$ upfront algorithm, $0.3<\mathrm{E}(\mathrm{T})<0.7$ for the algorithm interim, $0<\mathrm{E}(\mathrm{T})<0.3$ for the latter algorithm.

Second, the convergence speed is fast or slow, can be obtained. Fast convergence is defined - the critical point (is 0.05), convergence - the slow critical point (take 0.01), when the fast $\Delta E(t)>0.05$ convergence, while convergence $0.01<\Delta E(t)<0.05$ is normal, and when $\Delta E(t)<0.01$ to slow convergence. 
Adjustment. $\rho$ realization by adjusting the connection weights, first rule of thumb, set the lower limit $\rho_{\min }$ (to 0.1 ), the upper limit value $\rho_{\max }(0.95)$ and single-step change in the value of $\Delta \rho$ (eg 0.01), noting that $\rho$ must be decreasing ( $\rho$ large value of volatile strong, positive feedback intensifies; $\rho$ small value volatile weak, strengthen negative feedback), thus initializing $\rho=\rho_{\max }$. As shown in Fig. 2, structure adjustment strategy.

For a running ant colony algorithm, its "algorithm Period" and "real convergence rate" can be found by the method of Section 3.2, "expect convergence rate" must be from slow to fast, which is due early hope algorithm, ant positive feedback plays a leading role, in order to discover the area containing the optimal solution, then inevitably slow convergence; and algorithms mid ant strength equal positive and negative feedback, negative feedback makes the ants one hand began to approach the optimal solution, on the other hand positive feedback ensures ants from local optimum, so the convergence rate at a reasonable speed; and finally in the late algorithm has identified the global optimum of the region, and a region excluding local optimal solution, negative feedback play a leading role in enabling the rapid convergence of the ant to the global optimal solution, and it is now the fastest convergence [8-10].

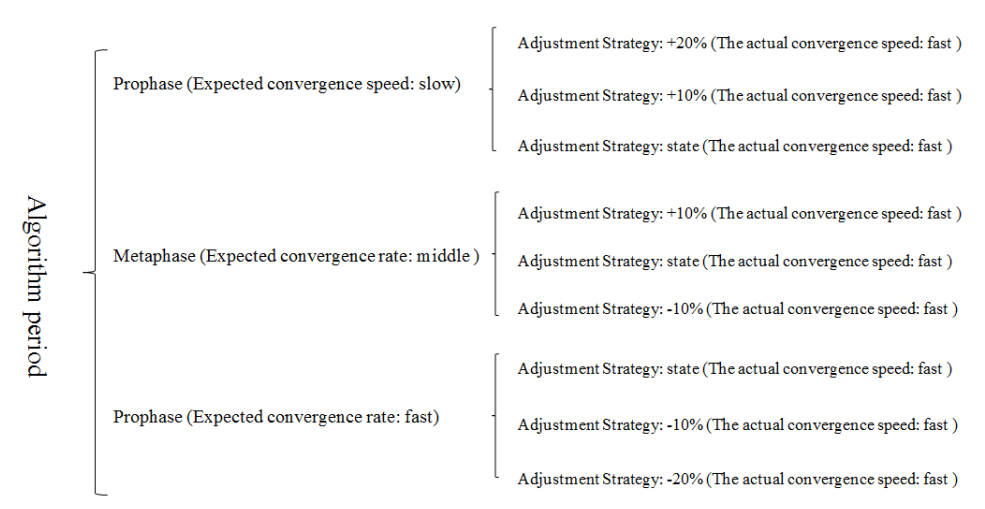

Figure 2. Strategy of parameter adjustment $(\rho)$

Wherein, variant $\rho$ has five kinds of "holding" means maintaining the status quo, "+ $10 \% "$ indicates an increase of $10 \% \rho, "+20 \% "$ indicates increased $20 \% \rho$; "- 10\%" indicates reduction $10 \% \rho$, "-20\% "indicates reduction $20 \% \rho$. This is because, when $\rho$ increases, pheromone evaporation is also enhanced, thus increasing the randomness algorithm convergence slow; when $\rho$ is reduced, pheromone evaporation is weakened, so deterministic algorithm to enhance convergence speed becomes faster.

\section{Case Analysis}

This paper selects domestic courier company four hundred thousand courier information as a test sample; wherein each city as shipping point node (test data a total of 5646 points freight). Among them, the transit time between the different shipping points; each express freight handling point of time; shipper freight shipped to the starting point used delivery time; the consignee to the final point of cargo receipt used time is; shipped from the consignor to the consignee receipt for the total time; then:

$$
T=t_{s}+\sum_{n=0}^{N} t_{n}+\sum_{m=0}^{M} t_{m}+t_{e}(n=0,1, \cdots, N ; m=0,1, \cdots, M)
$$

Ant colony algorithm and the improved ant colony algorithm optimization purposes time logistics route search is an optimal path that make $T$ minimum.

Specific parameter settings shown in Table 1, $\mathrm{n}$ must be consistent with the number of nodes, $\mathrm{m}$ 
select half of the number of nodes. $\rho$ default self-adaptation adjustment.

Table 1 Parameter setting

\begin{tabular}{|c|c|c|c|}
\hline$n$ & Nodes & $\rho_{\max }$ & 0.95 \\
\hline$m$ & $\mathrm{n} / 2$ & $\Delta \rho$ & 0.01 \\
\hline$\alpha$ & 2.0 & $T_{P M}$ & 0.7 \\
\hline$\beta$ & 4.2 & $T_{M A}$ & 0.3 \\
\hline$Q$ & 800 & $T_{F M}$ & 0.05 \\
\hline$\rho_{\min }$ & 0.1 & $T_{M S}$ & 0.01 \\
\hline
\end{tabular}

Convergence Performance Comparison. Improved ant colony algorithm and ant colony algorithm run 300 times, respectively, statistics converge to the global optimum number of convergence, as shown below:

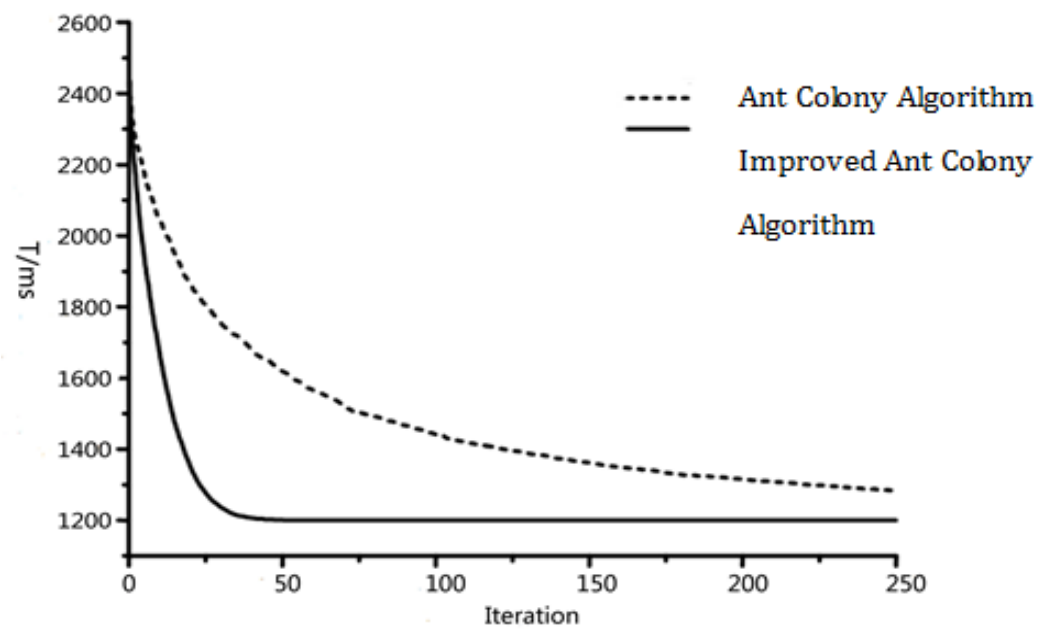

Figure 3. Ant colony algorithm and improved ant colony algorithm comparison chart

As can be seen from the chart, the positive and negative feedback regulation through improved ant colony algorithm connection weights than the traditional ant colony algorithm converges faster, and does not easily fall into the local optimal solution, the overall performance is improved.

\section{Conclusion}

Select time logistics route can achieve savings cargo transit time and improve operational efficiency, reduce operating costs and other important role. As used herein, the optimal solution based on the weights of positive and negative feedback ant colony algorithm search time logistics route; and, pre algorithm to adjust the parameters of positive feedback, avoiding falling into local optimal solution to ensure global optimal solution can be searched nearby, late negative feedback regulation algorithm parameters based, so that the ant colony can converge towards the global optimum. By way of example show that the proposed method is an effective way to solve the logistics, the algorithm can be used as the core business intelligence software logistics optimal path selection, it has a good prospect.

\section{References}

[1] KASTNER R. Synthesis techniques and optimizations for reconfigurable system [D]. Los Angeles: University of California, 2002. 
[2] SAHA D, MITRA R S, BASU A. Hardware software partitioning using genetic algorithm[C]. Proc. of the 10th Int'l Conf. on VLSI Design. Hyderabad: IEEE Computer Society Press, 1997: 155-160.

[3] GAJSKI D D, VAHID F, NARAYAN S, et al. Specsyn: An environment supporting the specify-explore-refine paradigm for hardware/software system design [J]. Read-ings in Hardware/Software Co-Design, 2002, 108- 124.

[4] ERNST R, HENKEL J, BENNER T. Hardware-software cosynthesis for microcontrollers [J]. IEEE Design \& Test of Computers, 1993, 10(4): 64-75.

[5] VAHID F, STITT G. Hardware/software partitioning [J]. Reconfigurable Computing, 2008: 539-560.

[6] F.M. Xu: Based On Evolution Ant Colony Algorithm TSP Research And Its Application [D]. (Jiangxi University Publications, China 2010).

[7] X.P. Wu and Y. Wu: Electronic Measurement and Instrument, Vol. 28 (2009) No4, p.105-111.

[8] L. Chen and L. Tong: Electronic Measurement and Instrument, Vol. 28 (2009) No5, p .147-151.

[9] Y.D. Zhang, L.N. Wu and W. G: Electronic Measurement and Instrument, Vol (2009) No8, p.32-38.

[10]C.F. Zhang: Computer and Information Technology, Vol. 26(2012) No3, p. 13-19. 